\title{
A cross-sectional study of the relationship between age and current health status for persons with spinal cord injuries
}

\author{
M J DeVivo DrPH, ${ }^{1} \mathrm{R}$ M Shewchuk $\mathrm{PhD},{ }^{2} \mathrm{~S}$ L Stover MD,${ }^{1} \mathrm{~K}$ J Black BS, ${ }^{1} \mathrm{~B}$ K Go \\ BA $^{1}$ \\ ${ }^{1}$ Department of Rehabilitation Medicine, ${ }^{2}$ Department of Health Services Administration, \\ University of Alabama at Birmingham, UAB Station, Birmingham, Alabama 35233-7330, \\ USA.
}

\begin{abstract}
As life expectancies of persons with spinal cord injuries increase, this population is aging rapidly. This trend requires that increasing attention be given to the healthcare needs of older persons with spinal cord injury. Follow up data on 11,117 persons injured since 1973 were analyzed by current age at 15 -year intervals. Mean time postinjury was 4.7 years. Several trends were observed when comparing persons currently in the 16-30 year age group with persons in the oldest age group (age 76+). The percentage of persons independent in selfcare decreased from $61.9 \%$ to $29.1 \%$. Ventilator use increased from $1.7 \%$ to $4.3 \%$. Nursing home residence increased from $1.4 \%$ to $22.2 \%$. The percentage of persons rehospitalized during the most recent year increased from $26.5 \%$ to $33.7 \%$. Age was an important predictor of health status, but time postinjury was less important, perhaps due to the study's short postinjury time frame. While few differences in health status were observed in individuals between 16 and 60 years of age, persons in the oldest two age groups demonstrated lower levels of health status than younger individuals.
\end{abstract}

Keywords: spinal cord injury; aging; health status; epidemiology; economics.

\section{Introduction}

Even though most spinal cord injuries occur among people who are relatively young, this population is aging rapidly as a result of increasing life expectancies. This trend requires that special attention be given to the healthcare needs of older people with spinal cord injuries.

We have previously published one comprehensive study and one abstract concerning the effect of age at time of injury on rehabilitation and short term treatment outcomes. $^{1,2}$ Although most published information on the long term effects of aging with a spinal cord injury is anecdotal in nature, a few studies have recently been conducted. $^{3-5}$ Nonetheless, there is a paucity of information in the literature concerning the current health status of older persons who were injured many years ago.

Recognizing this fact, the National Institute on Disability and Rehabilitation Research (NIDRR) recently established an absolute priority to fund a new Rehabilitation Research and Training Center (RRTC) on aging with a spinal cord injury. ${ }^{6}$ The first priority of this new RRTC will be to develop a better understanding of the natural course of spinal cord injury as persons age. This can only be accomplished by conducting more in-depth studies of the relationships between age, time since injury, and current health status, and comparing those findings to what would be expected in the absence of spinal cord injury. The present study attempts to accomplish that objective with a limited number of health status indicators by using data from the National Spinal Cord Injury Statistical Center database for persons injured since 1973 and followed annually thereafter with routine evaluations whenever possible for as many as 16 years.

\section{Methods}

The study population consisted of 11,117 persons with traumatic spinal cord injury 
occurring between 1973 and 1989 who were admitted to a model regional spinal cord injury care system within one year of injury. The place of injury and place of residence for all study population members were within the geographic catchment area of the model system. All persons had a clinically discernible degree of spinal cord neurological impairment on admission, were continually hospitalized from injury to model system admission except for brief periods no longer than normally accepted as a therapeutic leave of absence, and were discharged from the model system having completed rehabilitation.

Data from these persons were routinely collected prospectively on an annual basis and submitted to the National Spinal Cord Injury Statistical Center. A limited number of long term health status indicators were included in this national data set. These indicators were ventilator usage, place of residence, functional independence in selfcare and ambulation, and rehospitalizations during the preceding year.

For purposes of this study, ventilator usage was defined as either short term respiratory support as part of the medical treatment for other pulmonary complications, ventilator dependency, or placement of a phrenic nerve stimulator. Emergency mouth to mouth or machine resuscitation, routine administration of oxygen, emergency bagging, intermittent positive pressure breathing administration or postoperative ventilatory support used for less than 7 days did not qualify as ventilator usage.

Persons were considered functionally independent if they did not require any assistance in the performance of selfcare activities of daily living. To be considered ambulatory, persons must have been able to ambulate independently, and ambulation must have been the primary method of mobility.

Measures of injury severity such as neurological level of lesion and Frankel grade ${ }^{7}$ were also collected annually. A separate data set including demographics, acute care and rehabilitation medical information was collected at initial discharge from rehabilitation.
The data set was analyzed cross-sectionally using the most recent annual evaluation for each person. The average length of time postinjury for the annual evaluation used in this study was 4.7 years (range $1-16$ years).

All persons were divided into 6 age groups: age $1-15$ years $(n=123)$; age $16-30$ years $(n=5525)$; age $31-45$ years $(n=3405)$; age $46-60$ years $(n=1300)$; age 61-75 years $(n=632)$; age 76 and over $(n=132)$. The characteristics of each age group were expressed as percentages and the chi-square statistic was used to test the significance of observed differences among the age groups. ${ }^{8}$ Additional analyses were stratified by neurological level of lesion (paraplegia or quadriplegia) and by time since injury (more or less than 5 years).

Separate multiple logistic regression analyses were conducted for each outcome measure in order to control for possible confounding effects of sex, race, neurological level of lesion, Frankel grade, and ventilator usage. ${ }^{9}$ The adjusted odds ratio (OR) and its approximate $95 \%$ confidence interval $(\mathrm{CI})$ for each outcome were determined for each age group from the appropriate regression model coefficients. ${ }^{10}$ The odds ratio is an estimate of the likelihood of a given outcome among persons in each age group relative to the referent age group. For this study, the 16-30 year old age group was chosen as the referent group because the 1-15 year old age group was small in sample size and somewhat atypical of persons with spinal cord injury in general. An odds ratio of 1.0 implies no increased likelihood, whereas an odds ratio of 2.0 implies that persons in that age group were twice as likely to experience the particular outcome as were persons in the 16-30 year old age group. A 95\% confidence interval around an odds ratio that does not include 1.0 implies a statistically significant difference in the likelihood of an outcome for that age group at a two-tailed probability (alpha) of 0.05 .

\section{Results}

Demographic characteristics of the study population appear in Table I. The oldest age group had the highest percentage of whites 
Table I Characteristics (expressed as percentages) of each age group

\begin{tabular}{|c|c|c|c|c|c|c|c|}
\hline \multirow[b]{2}{*}{ Characteristic } & \multirow[b]{2}{*}{$1-15$} & \multirow[b]{2}{*}{$16-30$} & \multicolumn{2}{|c|}{ Age group (years) } & \multirow[b]{2}{*}{$61-75$} & \multirow[b]{2}{*}{$76+$} & \multirow[b]{2}{*}{$p$} \\
\hline & & & $31-45$ & $46-60$ & & & \\
\hline Male & 66.7 & 83.4 & 83.8 & 81.5 & 77.4 & 69.7 & $<.0001$ \\
\hline White & 66.7 & 75.4 & 72.0 & 67.1 & 71.4 & 79.5 & $<.0001$ \\
\hline Injury level & & & & & & & $<.0001$ \\
\hline $\mathrm{C} 1-\mathrm{C} 2$ & 14.0 & 1.3 & 1.2 & 1.7 & 2.0 & 2.1 & \\
\hline $\mathrm{C} 3-\mathrm{C} 4$ & 14.9 & 14.2 & 12.6 & 15.8 & 16.8 & 23.4 & \\
\hline $\mathrm{C} 5-\mathrm{C} 8$ & 18.4 & 38.5 & 35.5 & 34.7 & 40.0 & 45.7 & \\
\hline $\mathrm{T} 1-\mathrm{S} 5$ & 52.7 & 46.0 & 50.7 & 47.8 & 41.2 & 28.8 & \\
\hline Frankel grade & & & & & & & $<.0001$ \\
\hline Complete & 51.4 & 52.6 & 55.1 & 43.3 & 32.6 & 16.0 & \\
\hline $\begin{array}{l}\text { Preserved } \\
\text { sensation }\end{array}$ & 7.2 & 10.9 & 10.6 & 7.1 & 6.7 & 8.0 & \\
\hline $\begin{array}{l}\text { Motor } \\
\text { nonfunctional }\end{array}$ & 5.4 & 7.2 & 7.0 & 9.3 & 13.1 & 20.0 & \\
\hline Motor & & & & & & & \\
\hline functional & 33.3 & 26.3 & 26.2 & 38.6 & 44.3 & 55.0 & \\
\hline Recovered & 2.7 & 3.0 & 1.1 & 1.7 & 3.3 & 1.0 & \\
\hline
\end{tabular}

$(79.5 \%)$, persons with quadriplegia $(72.7 \%)$, and persons with neurologically incomplete motor functional injuries $(55.0 \%)$. The youngest and oldest age groups had lower percentages of males than the other age groups. These differences in demographic characteristics were all statistically significant $(p<0.0001)$ and, therefore, should be taken into consideration when interpreting the data on current health status of persons in each age group.

Measures of current health status appear in Table II. Except for the pediatric age group, the percentage of persons who were independent in selfcare activities of daily living decreased substantially with increasing age $(p<0.0001)$. Despite the fact that the oldest age group had the highest percentage of persons with neurologically incomplete motor functional injuries, only $29.1 \%$ of persons in that age group were considered independent. Among persons with paraplegia, the percentage who were independent decreased from $94 \%$ in the $16-30$ year old age group to $39.1 \%$ in the oldest age group $(p<0.0001)$. Among persons with quadriplegia, the trend was similar but less dramatic and not statistically significant, declining from $35 \%$ among persons in the $16-30$ year old age group to $26.2 \%$ in the oldest age group $(p>0.05)$. In large part, this is because the relationship between age and level of independence is confounded to a greater extent by differences in injury severity among persons with quadriplegia than among persons with paraplegia.

As expected, when the analysis was stratified by time postinjury, the percentage of younger persons (below age 46 years) who were independent decreased slightly with advancing time after injury. However, this trend was not observed among older persons, probably due to differential survival rates in the older age groups for persons who were and were not independent.

After controlling for differences in sex, race, neurological level of lesion, degree of injury completeness, and ventilator usage by using logistic regression analysis, persons in each successive age group were increasingly less likely to be independent in selfcare activities than the 16-30 year old age group $(p<0.05)$. In fact, persons in the oldest age group were only $16 \%$ as likely to be independent in selfcare activities as persons between the ages of 16 and 30 .

Older persons were slightly more likely to be ambulatory than younger persons because of the trend toward higher percentages of persons with incomplete motor 
Table II Health status measures (expressed as percentages and odds ratios) for each study population age group

\begin{tabular}{rcccccccc}
\hline & \multicolumn{7}{c}{ Age group (years) } & \\
Health status \\
indicator
\end{tabular}

functional injuries as age advanced $(p<0.0001)$. In fact, after controlling for differences in sex, race, neurological level of lesion, Frankel grade, and ventilator usage by using logistic regression analysis, no trend was observed in the odds ratios for independent ambulation for each age group.

There was a steady increase in the percentage of persons using ventilators beginning at age 16 that reached $4.3 \%$ in the oldest age group $(p<0.0001)$. Ventilator usage was rare among persons with paraplegia; while among persons with quadriplegia, ventilator usage increased from $3.1 \%$ among the 16-30 year olds to $6 \%$ in the oldest age group. Based on the results of the logistic regression analysis, persons in the oldest age group were 3.78 times more likely to require use of a ventilator than persons in the 16-30 year old age group $(p<0.05)$. Smaller but nonetheless statistically significant increases in the odds ratios for ventilator usage were also found for persons in the 61-75 year old age group and the 46-60 year old age group $(p<0.05)$. When the analysis was stratified by time after injury for persons of comparable age, ventilator usage was slightly more common during the first 5 years after injury, probably due to differential survival rates for ventilator users and nonusers.

Due to the high percentage of persons with $\mathrm{C} 1$ or $\mathrm{C} 2$ injury levels in the pediatric age group, ventilator usage was actually highest among persons in that group. However, even after controlling for differences in neurological level of lesion, Frankel grade, sex, and race by using logistic regression analysis, persons in the pediatric age group were still 2.82 times more likely to require use of a ventilator than persons between the ages of 16 and $30(p<0.05)$.

Most persons regardless of age were living 
in private residences. However, the percentage of persons living in private residences decreased steadily from $96.5 \%$ in the pediatric age group to $72.2 \%$ in the oldest age group $(p<0.0001)$. Conversely, the percentage of nursing home residents rose steadily from $0.9 \%$ in the pediatric age group to $22.2 \%$ in the oldest age group. In fact, among persons in the oldest age group who had quadriplegia, $25.3 \%$ resided in nursing homes. This compared with $13.3 \%$ of persons with paraplegia in that age group. After controlling for the confounding effects of sex, race, neurological level of lesion, Frankel grade, and ventilator usage, persons in the oldest age group were 22.2 times more likely to reside in nursing homes than their $16-30$ year old counterparts $(95 \% \mathrm{CI}$, 12.0-41.0). There were also statistically significant increases in the likelihood of nursing home residence for persons in the 61-75 year old age group (OR, 11.1; 95\% CI, 7.52-16.3), the 46-60 year old age group (OR, 3.05; 95\% CI, 2.03-4.56), and the 31-45 year old age group (OR, 1.64; $95 \% \mathrm{CI}, 1.16-2.32$ ) relative to persons between 16 and 30 years of age.

The pediatric age group contained the highest percentage of persons rehospitalized during the year $(37 \%)$. Excluding that age group, the percentage of rehospitalized persons increased steadily with advancing age from $26.5 \%$ in the $16-30$ year old age group to $33.7 \%$ in the oldest age group $(p<0.0001)$. Stratified analyses revealed that the percentage of persons who were rehospitalized was slightly higher during the first 5 years after injury than during later years for each age group. However, no meaningful differences in these percentages were observed between persons with paraplegia and persons with quadriplegia of comparable ages. Using multiple logistic regression analysis to control confounding due to relatively small sample sizes in the youngest and oldest age groups, only the 46-60 year old age group and the 61-75 year old age group had a significantly increased risk of rehospitalization compared to the 16-30 year olds $(p<0.05)$.

Many persons with spinal cord injuries experienced more than one rehospitalization during the year. When these multiple rehospitalizations were considered, the hospital discharge rate per thousand persons increased from 398 for persons between 16 and 45 years of age to 535 for persons over 75 years of age. Once again, because of the increased percentage of high level cervical injuries in the pediatric age group, it experienced the highest hospital discharge rate of 556 per thousand persons.

\section{Discussion}

This is the first study of which we are aware that has assessed broad measures of current health status in a large national sample of persons with relatively recent spinal cord injuries. Our study revealed that current age was an important predictor of health status following spinal cord injury, whereas time postinjury appeared to be considerably less important in most instances. A possible explanation for this finding could be the relatively short average time after injury for the study population.

In general, based on the indicators included in this study, the vast majority of persons with spinal cord injuries appear to be doing quite well. Almost everyone lived in a private residence, most were independent in performing selfcare activities of daily living, and few required ventilatory assistance.

While few significant differences were observed between 16 and 60 years of age, persons in the two oldest age groups demonstrated lower levels of health status than younger individuals. Persons in the oldest age groups were most likely to have cervical lesions and require the use of a ventilator. Perhaps our most striking findings were the high likelihood of nursing home residence in the two oldest age groups and the relatively low probability of independence in performing selfcare activities of daily living for those same age groups. In fact, a more detailed study of functional independence at discharge from rehabilitation by Yarkony et al yielded similar results. ${ }^{11}$ Interestingly, although rehospitalization rates for treatment of secondary medical complications were high for all age groups in our study population, they were not as strongly correlated 
with advancing age as was originally hypothesized.

Given the lower likelihood of functional independence for older persons with spinal cord injuries, it is not surprising that Krause and Crewe found lower activity levels, including reduced sitting tolerance, less frequency of leaving home on outings, and fewer weekly visitors, as well as less satisfaction with sex life and lower overall self-rated adjustment, as age increased. ${ }^{6}$ Moreover, these trends occurred despite the fact that their study was limited to persons no older than 65 years of age.

Given increasing life expectancies ${ }^{12}$ and the trend toward increasing age at injury, ${ }^{13}$ our findings, if confirmed, have implications that are important for the future of the model spinal cord injury care system program. The decline in independence levels that occurs with advancing age will inevitably result in increased burdens on an aging spouse and other family members. Numerous case studies have demonstrated that costs for attendant care, household assistance, and rehospitalizations may increase substantially. ${ }^{14,15}$

It should be noted that the associations between age and health status observed for our study population are very similar to those observed in the general population. Although the older population is heterogeneous with regard to health status, there is clearly an age associated decline in health. For example, among members of the general population residing in the community, the level of impairment also increases directly with age. However, for individuals of similar age, the proportion of persons who have impairment in their ability to perform activities of daily living is lower among members of the general population residing in the community than in our study population. The proportion of individuals in the general population having at least some difficulty in this area is about $10 \%$ for those between 65 and 69 years, $13 \%$ for those between 70 and 74 years, $20 \%$ for those between 75 and 79 years, 34\% for those between 80 and 84 years, and $57 \%$ for those over 85 years of age. ${ }^{16}$ By contrast, almost $71 \%$ of persons in our study population who were over 75 years of age were at least partially dependent in performing activities of daily living (Table II).

The use of nursing homes by the general population also tends to increase directly with advancing age. It is estimated that only about $1 \%$ of the general population between 65 and 74 years of age reside in a nursing home at any time; whereas about $6 \%$ of those between 75 and 85 years of age and about $22 \%$ of those over 85 years of age reside in nursing homes. ${ }^{17}$ By contrast, $12.4 \%$ of persons with spinal cord injuries between the ages of 61 and 75 and $22.2 \%$ of persons over 75 years of age reside in nursing homes (Table II). These figures suggest that persons with spinal cord injuries are more likely to enter nursing homes at an earlier age than members of the general population.

Similarly, hospital usage as measured by the number of discharged patients and days of care typically increases with age for persons in the general population. The discharged patient rate per thousand, as estimated by the National Center for Health Statistics, increases from approximately 157 for persons age 45 to 64 , to 281 for persons age 65 to 74 , to 427 for persons age 75 to 84 , and 533 for those 85 and older. ${ }^{18}$ By contrast, the discharged patient rate per thousand for the spinal cord injury population is considerably higher, reaching 453 for persons between 46 and 60 years of age, 524 for persons between 61 and 75 years of age, and 535 for persons over 75 years of age. The days of care provided by hospitals closely parallel the age trend noted for discharged patients. In general, older persons account for a disproportionate amount of hospital use. The American Hospital Association has noted that although older persons make up only about $12 \%$ of the population, approximately $25 \%$ of all hospital admissions are for persons over age 65 and that half of those admitted to hospitals are readmitted within a 12 month period. ${ }^{19}$

The model systems have long advocated the benefits of a routine annual follow up evaluation for most persons with spinal cord injuries. Given the findings of our study, more frequent return visits for older individuals should be encouraged, Moreover, we must develop more cost-effective service 
delivery mechanisms to meet the needs of elderly persons with spinal cord injuries. ${ }^{14}$ This might include more frequent use of public health nurses for in-home visits, cooperative living arrangements, enhanced linkages with independent living centers and an increased focus of their services toward problems associated with aging, and development of viable alternatives to nursing home placement such as lower cost intermediate care facilities.

We interpreted our findings cautiously for several reasons. First, the number of persons in the youngest and oldest age groups was not exceptionally large. Nonetheless, these age groups contained more than 100 persons each. Second, no attempt has been made to assess the causal nature of the relationship between age and these health status indicators. It is possible that some of these relationships are confounded by factors not included in the logistic regression models. However, additional confounding would occur only if an unincluded variable were related to both age and the health status indicator. Some potential confounding variables not included in this study are marital and financial status, insurance coverage, and comorbidities not related to the spinal cord injury. For example, although a general deterioration of health secondary to old age might cause nursing home placement, it is also possible that older persons were more likely to reside in nursing homes because they were more likely to live alone and to be without sufficient resources that would permit them to remain in their home. Other possible explanations could be that they were more likely to have had third party coverage of nursing home expenses, or they were more likely to have had other conditions independent of their spinal cord injury that required nursing home care. Even if advancing age is only a marker variable and not the actual cause of these changes in health status, it is still predictive of them and therefore can be useful for projecting future needs of the spinal cord injury population.

An additional issue of concern is that this study did not involve a random sample of individuals. Since many individuals are typically lost to follow up, it is possible that these results are somewhat biased toward poorer health status because persons who have problems are more likely to return for routine annual evaluations. This potential bias may be compounded by the fact that persons initially treated at model systems are on average more severely injured than persons initially treated elsewhere. However, these potential biases may be offset, at least partially, by this study population's participation in the organized multidisciplinary follow up program of the model systems. These programs are designed to prevent the occurrence of secondary complications that might lead to rehospitalization or nursing home stays. Therefore, additional research is needed to confirm our results with either a prospective long term follow up study or a similar cross-sectional study based on a randon sample of individuals with more elapsed time after injury.

\section{Acknowledgements}

Preparation of this manuscript was supported in part by grant \#H133N00001 from the National Institute on Disability and Rehabilitation Research (NIDRR), Department of Education, Washington, DC, USA.

The following model regional spinal cord injury care systems contributed data to the National Spinal Cord Injury Statistical Center data set that was used in this study: University of Alabama at Birmingham, Birmingham, AL; Good Samaritan Medical Center and Barrow Neurological Institute, St. Joseph's Hospital, Phoenix, AZ; Santa Clara Valley Medical Center, San Jose, CA; Rancho Los Amigos Medical Center, Downey, CA; Craig Hospital, Englewood, CO; University of Miami School of Medicine, Miami, FL; Shepherd Center for Treatment of Spinal Injuries, Atlanta, GA; Northwestern Memorial Hospital and Rehabilitation Institute of Chicago, Chicago, IL; Louisiana State University Medical Center, New Orleans, LA; Boston University Medical Center, Boston, MA; Rehabilitation Institute of Michigan, Detroit, MI; University of Missouri School of Medicine, Columbia, MO; Rusk Institute of Rehabilitation Medicine, New York University Medical Center, New York, NY; University of Rochester Medical Center, Rochester, NY; Jefferson Medical College, Thomas Jefferson University, Philadelphia, PA; The Institute for Rehabilitation Research, 
Houston, TX; University of Virginia Medical Center, Charlottesville, VA; University of
Washington, Seattle, WA; University of Michigan Medical Center, Ann Arbor, MI.

\section{References}

1 DeVivo MJ, Kartus PL, Rutt RD, Stover SL, Fine PR (1990) The influence of age at time of spinal cord injury on rehabilitation outcome. Arch Neurol 47: 687-691.

2 DeVivo MJ, Black KJ, Go BK, Stover SL (1990) Effect of age on rehabilitation outcomes of spinal cord injury patients. Arch Phys Med Rehabil 71: 787.

3 Krause JS, Crewe NM (1991) Chronologic age, time since injury, and time of measurement: effect on adjustment after spinal cord injury. Arch Phys Med Rehabil 72: 91-100.

4 Whiteneck GG, Charlifue SW, Gerhart KA, Lammertse DP, Manley S, Menter RR et al editors (in press) Aging with Spinal Cord Injury. Demos Publications, New York.

5 Whiteneck GG, Charlifue SW, Frankel HL, Fraser MH, Gardner BP, Gerhart KA et al (in press) Mortality, morbidity, and psychosocial outcomes of persons spinal cord injured more than 20 years ago. Paraplegia.

6 Federal Register (1992) July 6, 57 (129): 29766-29772.

7 Frankel HL, Hancock DO, Hyslop G, Melzak J, Michaelis L, Ungar G et al (1969) The value of postural reduction in the initial management of closed injuries of the spine with paraplegia and tetraplegia. Paraplegia 7(part 1): 179-192.

8 Fleiss JL (1973) Statistical Methods for Rates and Proportions. John Wiley \& Sons Inc, New York: 92-108.

9 Kleinbaum DG, Kupper LL, Morgenstern H (1982) Epidemiologic Research: Principles and Quantitative Methods. Lifetime Learning Publications, Belmont, CA: 419-491.

10 Miettinen O (1976) Estimability and estimation in case-referent studies. Am J Epidemiol 103: 226-235.

11 Yarkony GM, Roth EJ. Heinemann AW, Lovell LL (1988) Spinal cord injury rehabilitation outcome: the impact of age. J Clin Epidemiol 41: 173-177.

12 DeVivo MJ, Stover SL, Black KJ (1992) Prognostic factors for 12-year survival after spinal cord injury. Arch Phys Med Rehabil 73: 156-162.

13 DeVivo MJ, Rutt RD, Black KJ, Go BK, Stover SL (1992) Trends in spinal cord injury demographics and treatment outcomes between 1973 and 1986. Arch Phys Med Rehabil 73: 424-430.

14 Trieschmann RB (1987) Aging with a Disability. Demos Publications, New York: 128-132.

15 Menter RR (1989) Aging and spinal cord injury: implications for existing model systems and future federal, state, and local health care policy In: Spinal Cord Injury: The Model. Proceedings of the National Consensus Conference on Catastrophic Illness and Injury, December, 1989. Shepherd Center for Treatment of Spinal Injuries, Inc, Atlanta, GA: 72-80.

16 (1991) Aging America: Trends and Projections.

17 Hing E (1987) Use of nursing homes by the elderly: preliminary data from the national nursing home survey. Advance Data No. 135, National Center for Health Statistics.

18 National Center for Health Statistics (1989) National Hospital Discharge Survey: Annual Summary, 1987. Vital and Health Statistics Series 13 No. 99.

19 AHA Report (1989) Hospitals and Older Adults: Meeting the Challenge. American Hospital Association, Chicago, IL. 